\title{
The relationship of fibroblast growth factor 21 with cardiovascular outcome events in the Fenofibrate Intervention and Event Lowering in Diabetes study
}

\author{
Kwok-Leung Ong • Andrzej S. Januszewski • Rachel O'Connell • Alicia J. Jenkins • \\ Aimin Xu • David R. Sullivan • Philip J. Barter • Wei-Ting Hung • Russell S. Scott • \\ Marja-Riitta Taskinen • Anthony C. Keech • Kerry-Anne Rye
}

Received: 14 July 2014 / Accepted: 5 November 2014 /Published online: 26 November 2014

(C) Springer-Verlag Berlin Heidelberg 2014

\begin{abstract}
Aims/hypothesis Circulating fibroblast growth factor 21 (FGF21) levels are often elevated in obesity, dyslipidaemia, insulin resistance and type 2 diabetes. This study investigated the relationship of plasma FGF21 levels with cardiovascular events in patients with type 2 diabetes.

Methods Plasma FGF21 levels were measured by ELISA at baseline in 9,697 individuals with type 2 diabetes participating in the Fenofibrate Intervention and Event Lowering in Diabetes (FIELD) study. We assessed the association of FGF21
\end{abstract}

Anthony C. Keech and Kerry-Anne Rye are joint senior authors.

Electronic supplementary material The online version of this article (doi:10.1007/s00125-014-3458-7) contains peer-reviewed but unedited supplementary material, which is available to authorised users.

K.-L. Ong • P. J. Barter $\cdot$ K.-A. Rye

Lipid Research Group, Heart Research Institute, Sydney, NSW,

Australia

K.-L. Ong • P. J. Barter $\cdot$ K.-A. Rye

Faculty of Medicine, University of Sydney, Sydney, NSW, Australia

K.-L. Ong $(\varangle) \cdot$ P. J. Barter $\cdot$ K.-A. Rye

Centre for Vascular Research, University of New South Wales,

Sydney, NSW 2052, Australia

e-mail: oklws@yahoo.com.hk

K.-L. Ong • A. S. Januszewski • R. O'Connell • A. J. Jenkins •

W.-T. Hung $\cdot$ A. C. Keech

National Health and Medical Research Council Clinical Trials

Centre, University of Sydney, Sydney, NSW, Australia

A. S. Januszewski $\cdot$ A. J. Jenkins

Department of Medicine, University of Melbourne, Melbourne, VIC, Australia

\section{A. $\mathrm{Xu}$}

Department of Medicine, University of Hong Kong,

Hong Kong SAR, People's Republic of China levels with the incidence of different cardiovascular outcomes over 5 years. The primary outcome was total cardiovascular disease (CVD) events and the secondary outcomes were the four individual components: coronary heart disease events, total stroke, CVD mortality and coronary and carotid revascularisation. The tertiary outcome was hospitalisation for angina pectoris.

Results Higher baseline FGF21 levels were associated with higher risks of all cardiovascular outcome events after adjusting for the study treatment allocation (all $p<0.01$ ). The

A. $\mathrm{Xu}$

State Key Laboratory of Pharmaceutical Biotechnology, University of Hong Kong, Hong Kong SAR, People's Republic of China

D. R. Sullivan

Department of Clinical Biochemistry, Royal Prince Alfred Hospital, Sydney, NSW, Australia

\section{R. S. Scott}

Lipid and Diabetes Research Group, Christchurch Hospital, Christchurch, New Zealand

M.-R. Taskinen

Heart and Lung Centre, Cardiovascular Research Unit, Helsinki University Central Hospital, Helsinki, Finland

M.-R. Taskinen

Diabetes and Obesity Research Program, University of Helsinki, Biomedicum, Helsinki, Finland

\section{A. C. Keech}

Department of Cardiology, Royal Prince Alfred Hospital, Sydney, NSW, Australia 
associations remained significant for total CVD events and for coronary and carotid revascularisation after further adjusting for confounding factors, with the HR $(95 \% \mathrm{CI})$ being 1.28 $(1.10,1.50)$ and $1.26(1.01,1.56)$, respectively, for the highest tertile compared with the lowest tertile (overall effect $p=0.002$ and 0.007 , respectively). The addition of FGF21 levels to a model including established CVD risk factors predicting total CVD events led to a non-significant increase in the C-statistic but there was a significant improvement in integrated discrimination and net reclassification.

Conclusions/interpretation Higher baseline plasma FGF21 levels were associated with higher risk of cardiovascular events in patients with type 2 diabetes.

Trial registration: ISRCTN64783481

Keywords Cardiovascular outcome · Fenofibrate . Fenofibrate Intervention and Event Lowering in Diabetes (FIELD) study · Fibroblast growth factor 21

$\begin{array}{ll}\text { Abbreviations } \\ \text { CHD } & \text { Coronary heart disease } \\ \text { CVD } & \text { Cardiovascular disease } \\ \text { FGF21 } & \text { Fibroblast growth factor } 21 \\ \text { FIELD } & \text { Fenofibrate Intervention and Event Lowering in } \\ & \text { Diabetes } \\ \text { IDI } & \text { Integrated discrimination improvement } \\ \text { IQR } & \text { Interquartile range } \\ \text { NRI } & \text { Net reclassification improvement }\end{array}$

\section{Introduction}

Fibroblast growth factor 21 (FGF21) is a novel metabolic regulator [1-4]. In animal studies, FGF21 was found to have anti-inflammatory, hypoglycaemic and hypolipidaemic effects $[1,5,6]$. However, in human studies, circulating FGF21 levels are often elevated in obesity, dyslipidaemia, insulin resistance, type 2 diabetes, non-alcoholic fatty liver disease and coronary artery disease [4, 7-10]. The elevation in FGF21 levels may be a compensatory response to underlying metabolic stress or may be due to impaired FGF21 signalling leading to FGF21 resistance [4]. In recent years, several pharmaceutical companies have carried out pre-clinical studies to investigate the benefits of FGF21-based therapies in diabetes and obesityassociated disorders [11-13]. In a recent proof-of-concept trial, daily subcutaneous injection of a variant of FGF21 resulted in favourable changes in lipid levels, lipoprotein profile, body weight, fasting insulin and adiponectin levels in obese patients with type 2 diabetes [14]. Recent studies in animals have also demonstrated that FGF21 has a cardioprotective effect $[15,16]$.
The aim of the Fenofibrate Intervention and Event Lowering in Diabetes (FIELD) study was to investigate the effect of long-term lipid-lowering treatment with fenofibrate on adverse macrovascular outcomes in patients with type 2 diabetes [17]. In this study, although fenofibrate therapy did not significantly reduce the primary outcome of major coronary events over 5 years, it did reduce the main pre-specified secondary endpoint of total cardiovascular disease (CVD) events in patients with type 2 diabetes [18]. There are very limited data in the literature on the relationship of circulating FGF21 levels with CVD events in a large-scale, well-designed cohort. The size of the FIELD trial $(n=9,795)$ and number of cardiovascular events provide a unique opportunity to examine the relationship of FGF21 levels to cardiovascular risk. Therefore, in this 5 year study, we investigated whether circulating FGF21 levels were associated with any cardiovascular outcome events, and whether FGF21 levels could provide incremental information on risk stratification compared with a model using traditional cardiovascular risk factors alone.

\section{Methods}

Study design The study design, baseline characteristics of the participants and major findings of the FIELD study have been described previously [17-21]. Briefly, the FIELD study (International Standard Randomised Controlled Trial [ISRCTN] registration no. 64783481) was a double-blind placebo-controlled randomised trial, involving a total of 9,795 patients with type 2 diabetes recruited into the study between February 1998 and November 2000. All patients were aged 50-75 years and were randomly allocated to receive once-daily comicronised fenofibrate $200 \mathrm{mg}$ or matching placebo for 5 years. The study protocol was approved by national and local ethics committees. The study was undertaken in accordance with the Declaration of Helsinki and Good Clinical Practice Guidelines. All study participants gave written consent.

Plasma FGF21 measurement In the present study, 9,697 $(99.0 \%)$ out of 9,795 patients from the FIELD study had plasma samples at baseline available for FGF21 measurement. In a separate analysis, plasma FGF21 levels were also measured in a random subsample of 1,919 patients $(62.4 \%$ men; $49.8 \%$ in the fenofibrate treatment group) at 1 year to assess the effect of fenofibrate treatment. FGF21 levels were measured using ELISA kits (Antibody and Immunoassay Services, University of Hong Kong, Hong Kong SAR, People's Republic of China) as described previously in our pilot study [22]. Briefly, a $60 \mu$ l plasma sample was diluted 1:1 (vol./vol.) with assay diluent and analysed together with quality controls according to manufacturer's instructions. The intra-assay and inter-assay coefficients of variation were $<6 \%$. All samples 
were analysed masked for patient identity and study treatment allocation.

Other variables of interest The methods for measuring clinical characteristics, and details on the primary endpoint and other outcomes of the FIELD trial, have been described previously [17-24]. In this analysis, as specified for all FIELD biomarker analyses, the primary outcome was total CVD events (coronary heart disease [CHD] events, total stroke and other cardiovascular death events plus coronary and carotid revascularisation). The secondary outcomes in this analysis were the individual components of total CVD events (i.e. CHD event, total stroke, CVD mortality and coronary and carotid revascularisation). In this study, we also analysed the tertiary outcome of hospital admission for angina pectoris, which included unstable angina, other forms of angina pectoris and unspecified angina pectoris with matched ICD10 codes of I20.0, I20.8 and I20.9 (www.who.int/ classifications/icd/en/). In an exploratory analysis, the outcomes of CHD events, total stroke and CVD mortality were combined as the hard endpoint. Previous CVD comprised angina, myocardial infarction, coronary artery bypass grafting, percutaneous transluminal coronary angioplasty, stroke, peripheral vascular disease and revascularisation. Estimated GFR was calculated by the four-variable Modification of Diet in Renal Disease formula [25].

Statistical analysis Statistical analysis was performed using SPSS 22 (IBM, Armonk, NY, USA) or STATA 13.0 (StataCorp, College Station, TX, USA). The relative change in FGF21 level from baseline to 1 year was calculated after $\log _{\mathrm{e}}$ transformation (i.e. the average difference in $\log _{\mathrm{e}}$-transformed FGF21 level was exponentiated to obtain a ratio, which was rescaled by subtracting 1 and multiplying by 100 to derive the relative change). Treatment effect was derived from the ratio of treatment-specific ratios for the fenofibrate group relative to the placebo group. Cox regression was used to compute HR and its $95 \%$ CIs to assess the association of FGF21 levels at baseline with different outcome events. As FGF21 level was highly skewed, data were analysed after $\log _{\mathrm{e}}$ transformation to achieve a more linear relationship with outcomes. Tests for deviation from linearity indicated a significant deviation from linearity for the outcome of coronary and carotid revascularisation, but not for other outcomes [26]. Therefore, tertiles of baseline FGF21 levels were used as the main analysis. The $p$ value for the overall effect of FGF21 tertile was calculated by testing the hypothesis that the HR estimates of both tertiles 2 and 3 were equal to one. The incremental value of the addition of $\log _{\mathrm{e}}$-transformed FGF21 levels in the risk prediction model was assessed by the change in Harrell's C-statistic using a method adapted for survival models, integrated discrimination improvement (IDI) and the category-free net reclassification improvement (NRI), as described previously [27-29]. The goodness of fit of the models was assessed using the Gronnesby and Borgan test [30]. See the online electronic supplementary material (ESM) Methods for further details.

In this analysis, the principal pre-specified analysis was the association of baseline FGF21 levels with different outcomes in Cox regression analysis. A two-sided $p$ value $<0.05$ was considered significant for the primary outcome, $p<0.01$ for the secondary outcomes and $p<0.001$ for the tertiary outcome. For the post hoc analysis of the association of FGF21 levels with hard endpoint, and the subgroup analysis of total CVD events, a $p$ value $<0.01$ was considered significant. A twosided $p$ value $<0.05$ was considered significant for all other analyses.

\section{Results}

Baseline characteristics There were no significant differences in age, sex, BMI, waist-to-hip ratio, known diabetes duration or percentage of prior CVD between the 9,697 patients with available baseline FGF21 measurements and the other 98 patients without measurements (all $p>0.05$ ) (ESM Table 1). The median value (interquartile range [IQR]) of baseline plasma FGF21 was $316(205-482) \mathrm{pg} / \mathrm{ml}$. Table 1 shows the clinical characteristics of all the patients according to tertiles of FGF21 levels at baseline.

Effect of fenofibrate therapy on FGF21 level Over 1 year, plasma FGF21 levels increased by about $101.5 \%$ in the fenofibrate group, but only by $11.2 \%$ in the placebo group, compared with baseline levels. Fenofibrate increased plasma FGF 21 levels by $81 \%(95 \%$ CI 72,90$)$ relative to placebo over 1 year $(p<0.001)$. Although women had higher FGF21 levels than men at baseline and after 1 year (all $p<0.01$ ), the effect of fenofibrate therapy on relative change in plasma FGF21 was similar in men and women (ESM Table 2).

Association of FGF21 level with baseline characteristics As shown in ESM Table 3, higher age, BMI, waist-to-hip ratio, fasting glucose, HOMA-IR, triacylglycerol and plasma creatinine and homocysteine, as well as female sex, smoking and prior history of CVD, were independently associated with higher FGF21 levels, while longer known diabetes duration and higher LDL-cholesterol, $\mathrm{HbA}_{1 \mathrm{c}}$ and fibrinogen were associated with lower FGF21 levels. In a separate analysis, the effect of concomitant use of baseline glucose-lowering and cardiovascular medications on FGF21 levels was analysed. For glycaemic control, compared with diet therapy alone, the use of oral agents (mainly metformin and/or a sulfonylurea) was associated with higher FGF21 levels ( $p=0.009$ ), whereas 
Table 1 Characteristics of patients at baseline grouped according to baseline plasma FGF21 level

\begin{tabular}{|c|c|c|c|}
\hline Characteristic & Tertile $1(<239.3 \mathrm{pg} / \mathrm{ml})$ & Tertile $2(239.3$ to $<412.8 \mathrm{pg} / \mathrm{ml})$ & Tertile $3(\geq 412.8 \mathrm{pg} / \mathrm{ml})$ \\
\hline$n$ & 3,232 & 3,233 & 3,232 \\
\hline FGF21 (pg/ml) & $170(127-205)$ & $316(276-360)$ & $585(482-786)$ \\
\hline Age (years) & $61.8 \pm 6.8$ & $62.3 \pm 6.9$ & $62.6 \pm 6.9$ \\
\hline Male sex & $2,247(69.5 \%)$ & $2,044(63.2 \%)$ & $1,787(55.3 \%)$ \\
\hline White ethnicity & $3,031(94.1 \%)$ & $3,044(94.4 \%)$ & $3,067(95.1 \%)$ \\
\hline Current smoker & $262(8.1 \%)$ & $281(8.7 \%)$ & $372(11.5 \%)$ \\
\hline Ex-smoker & $1,590(49.2 \%)$ & $1,649(51.0 \%)$ & $1,655(51.2 \%)$ \\
\hline Prior history of CVD & $580(17.9 \%)$ & $700(21.7 \%)$ & $822(25.4 \%)$ \\
\hline Known diabetes duration (years) & $6(3-10)$ & $5(2-10)$ & $5(2-9)$ \\
\hline BMI $\left(\mathrm{kg} / \mathrm{m}^{2}\right)$ & $28.5(25.8-31.8)$ & $30.0(27.0-33.5)$ & $31.1(27.9-35.4)$ \\
\hline Waist-to-hip ratio & $0.93(0.88-0.98)$ & $0.94(0.89-0.98)$ & $0.94(0.88-0.99)$ \\
\hline Fasting insulin (pmol/l) & $60(37-90)$ & $72(48-108)$ & $90(60-132)$ \\
\hline Fasting glucose (mmol/l) & $8.3(6.9-10.1)$ & $8.4(7.0-10.2)$ & $8.7(7.2-10.6)$ \\
\hline $\mathrm{HbA}_{1 \mathrm{c}}(\%)$ & $6.8(6.1-7.8)$ & $6.8(6.1-7.7)$ & $7.0(6.2-7.9)$ \\
\hline $\mathrm{HbA}_{1 \mathrm{c}}(\mathrm{mmol} / \mathrm{mol})$ & $50.8(42.6-61.2)$ & $50.8(42.6-60.7)$ & $52.5(43.7-62.8)$ \\
\hline HOMA-IR & $1.44(0.97-2.17)$ & $1.76(1.17-2.58)$ & $2.16(1.47-3.13)$ \\
\hline Systolic BP (mmHg) & $139 \pm 15$ & $141 \pm 15$ & $142 \pm 15$ \\
\hline Diastolic BP (mmHg) & $82 \pm 9$ & $82 \pm 8$ & $82 \pm 8$ \\
\hline Total cholesterol (mmol/l) & $5.00 \pm 0.68$ & $5.06 \pm 0.70$ & $5.05 \pm 0.72$ \\
\hline HDL-cholesterol (mmol/l) & $1.13 \pm 0.27$ & $1.09 \pm 0.25$ & $1.07 \pm 0.25$ \\
\hline LDL-cholesterol (mmol/l) & $3.12 \pm 0.62$ & $3.09 \pm 0.65$ & $2.99 \pm 0.68$ \\
\hline Triacylglycerol (mmol/l) & $1.50(1.20-1.95)$ & $1.76(1.38-2.30)$ & $1.99(1.55-2.69)$ \\
\hline Apolipoprotein A-I (g/l) & $1.30 \pm 0.21$ & $1.29 \pm 0.21$ & $1.29 \pm 0.21$ \\
\hline Apolipoprotein A-II (g/l) & $0.35 \pm 0.07$ & $0.35 \pm 0.07$ & $0.35 \pm 0.07$ \\
\hline Apolipoprotein B (g/l) & $0.95 \pm 0.16$ & $0.98 \pm 0.17$ & $0.99 \pm 0.18$ \\
\hline Fibrinogen (g/l) & $3.58 \pm 0.71$ & $3.60 \pm 0.76$ & $3.60 \pm 0.77$ \\
\hline Plasma creatinine $(\mu \mathrm{mol} / \mathrm{l})$ & $77.1 \pm 14.6$ & $77.3 \pm 15.7$ & $78.4 \pm 16.9$ \\
\hline Homocysteine $(\mu \mathrm{mol} / \mathrm{l})$ & $9.2(7.8-11.1)$ & $9.5(8.0-11.3)$ & $10.0(8.2-12.2)$ \\
\hline Estimated GFR $\left(\mathrm{ml} \mathrm{min}{ }^{-1} 1.73 \mathrm{~m}^{-2}\right)$ & $89.7 \pm 17.5$ & $88.2 \pm 18.4$ & $85.2 \pm 19.0$ \\
\hline
\end{tabular}

Data are expressed as mean $\pm \mathrm{SD}, n(\%)$ or median (IQR)

the use of insulin was associated with lower FGF21 levels $(p<0.001)$ (ESM Table 4). For cardiovascular medication, the use of aspirin was associated with lower FGF21 levels $(p=0.02)$, whereas the use of diuretic was associated with higher FGF21 levels $(p<0.001)$ (ESM Table 5).

Association of baseline FGF21 level with cardiovascular events Of the 9,697 patients with valid data on FGF21 levels, 1,281 developed total CVD events, 537 developed CHD events, 330 developed stroke, 266 developed CVD mortality, 691 developed coronary and carotid revascularisation and 455 needed hospitalisation for angina pectoris during a median follow-up period of 5 years. Table 2 shows the association of tertiles of plasma FGF21 levels at baseline with different outcome events over 5 years. When only adjusting for treatment allocation, higher tertiles of plasma FGF21 levels were associated with higher risks of all outcome events (all overall effect $p<0.01)$. However, the association remained significant only for total CVD events $(p=0.002)$ and for coronary and carotid revascularisation $(p=0.007)$ after adjusting for confounding factors (Table 2 and Fig. 1a). Associations were demonstrated for total stroke and hospitalisation for angina pectoris although they did not meet the pre-specified criteria for a 'significant' $p$ value for secondary and tertiary outcomes. Figure $1 \mathrm{~b}$ shows the cumulative first event rates over 5 years by tertile of baseline FGF21 levels for all the outcomes. A significant interaction with treatment allocation was found for total CVD events, in which the association was significant in the placebo group, but not the fenofibrate group (overall effect $p<0.001$ and $p=0.19$, respectively; $p$ for interaction $=0.04$ ) (Table 2). In a separate analysis, similar results were obtained for the association of FGF21 tertiles with different outcome events after further adjusting for glucose-lowering medication, aspirin and diuretic (FGF21 tertile overall effect: total 
Table 2 Association of plasma FGF21 level at baseline with outcome events over 5 years
For all the outcomes, tertile 1 was the reference group used for comparison

${ }^{\text {a }}$ Adjusted for treatment allocation

${ }^{\mathrm{b}}$ Further adjusted for age, sex, known diabetes duration, prior history of CVD, smoking (never, former and current), $\mathrm{BMI}, \mathrm{HbA}_{1 \mathrm{c}}$, HOMA-IR, systolic BP, HDLcholesterol, LDL-cholesterol, triacylglycerol, fibrinogen, plasma creatinine and homocysteine at baseline

\begin{tabular}{|c|c|c|}
\hline Outcome & $\begin{array}{l}\text { HR }(95 \% \text { CI }) \\
\text { Model } 1^{\text {a }}\end{array}$ & Model $2^{\mathrm{b}}$ \\
\hline \multicolumn{3}{|l|}{ Primary outcome } \\
\hline \multicolumn{3}{|l|}{ Total CVD events in all participants } \\
\hline Tertile 2 & $1.35(1.17,1.55)$ & $1.28(1.10,1.48)$ \\
\hline Tertile 3 & $1.45(1.27,1.67)$ & $1.28(1.10,1.50)$ \\
\hline Overall effect $p$ value & $<0.001$ & 0.002 \\
\hline$p$ value for treatment interaction & 0.07 & 0.04 \\
\hline \multicolumn{3}{|c|}{ Total CVD events in participants receiving placebo } \\
\hline Tertile 2 & $1.55(1.27,1.88)$ & $1.52(1.24,1.88)$ \\
\hline Tertile 3 & $1.49(1.23,1.81)$ & $1.35(1.08,1.69)$ \\
\hline Overall effect $p$ value & $<0.001$ & $<0.001$ \\
\hline \multicolumn{3}{|c|}{ Total CVD events in participants receiving fenofibrate } \\
\hline Tertile 2 & $1.15(0.94,1.41)$ & $1.04(0.84,1.30)$ \\
\hline Tertile 3 & $1.42(1.17,1.72)$ & $1.21(0.97,1.51)$ \\
\hline Overall effect $p$ value & 0.002 & 0.19 \\
\hline \multicolumn{3}{|l|}{ Secondary outcomes } \\
\hline \multicolumn{3}{|l|}{ CHD event } \\
\hline Tertile 2 & $1.29(1.04,1.61)$ & $1.16(0.92,1.47)$ \\
\hline Tertile 3 & $1.60(1.29,1.98)$ & $1.23(0.97,1.57)$ \\
\hline Overall effect $p$ value & $<0.001$ & 0.23 \\
\hline$p$ value for treatment interaction & 0.66 & 0.48 \\
\hline \multicolumn{3}{|l|}{ Total stroke } \\
\hline Tertile 2 & $1.47(1.10,1.95)$ & $1.39(1.02,1.89)$ \\
\hline Tertile 3 & $1.75(1.32,2.30)$ & $1.48(1.08,2.03)$ \\
\hline Overall effect $p$ value & $<0.001$ & 0.04 \\
\hline$p$ value for treatment interaction & 0.47 & 0.30 \\
\hline \multicolumn{3}{|l|}{ CVD mortality } \\
\hline Tertile 2 & $1.32(0.96,1.82)$ & $1.11(0.79,1.57)$ \\
\hline Tertile 3 & $1.72(1.27,2.33)$ & $1.14(0.80,1.62)$ \\
\hline Overall effect $p$ value & 0.002 & 0.75 \\
\hline$p$ value for treatment interaction & 0.51 & 0.35 \\
\hline \multicolumn{3}{|l|}{ Coronary and carotid revascularisation } \\
\hline Tertile 2 & $1.43(1.18,1.72)$ & $1.37(1.12,1.67)$ \\
\hline Tertile 3 & $1.32(1.09,1.60)$ & $1.26(1.01,1.56)$ \\
\hline Overall effect $p$ value & $<0.001$ & 0.007 \\
\hline$p$ value for treatment interaction & 0.14 & 0.28 \\
\hline \multicolumn{3}{|l|}{ Tertiary outcome } \\
\hline \multicolumn{3}{|l|}{ Hospitalisation for angina pectoris } \\
\hline Tertile 2 & $1.62(1.27,2.07)$ & $1.42(1.10,1.84)$ \\
\hline Tertile 3 & $1.82(1.43,2.32)$ & $1.51(1.15,1.98)$ \\
\hline Overall effect $p$ value & $<0.001$ & 0.007 \\
\hline$p$ value for treatment interaction & 0.77 & 0.48 \\
\hline
\end{tabular}

CVD events, $p=0.002$; CHD event, $p=0.22$; total stroke, $p=0.04$; CVD mortality, $p=0.81$; coronary and carotid revascularisation, $p=0.006$; hospitalisation for angina pectoris, $p=0.006$ ). In an exploratory analysis, when the outcomes of CHD event, total stroke and CVD mortality were combined as a hard endpoint, the association of FGF21 levels did not reach the pre-specified $p$ value criteria for significance for post hoc analysis (ESM Table 6). Similar results were obtained when $\log _{\mathrm{e}}$-transformed FGF21 levels, instead of FGF21 tertiles, were used in the analysis although the interaction with treatment allocation was not significant for total CVD events (ESM Table 7). 
a

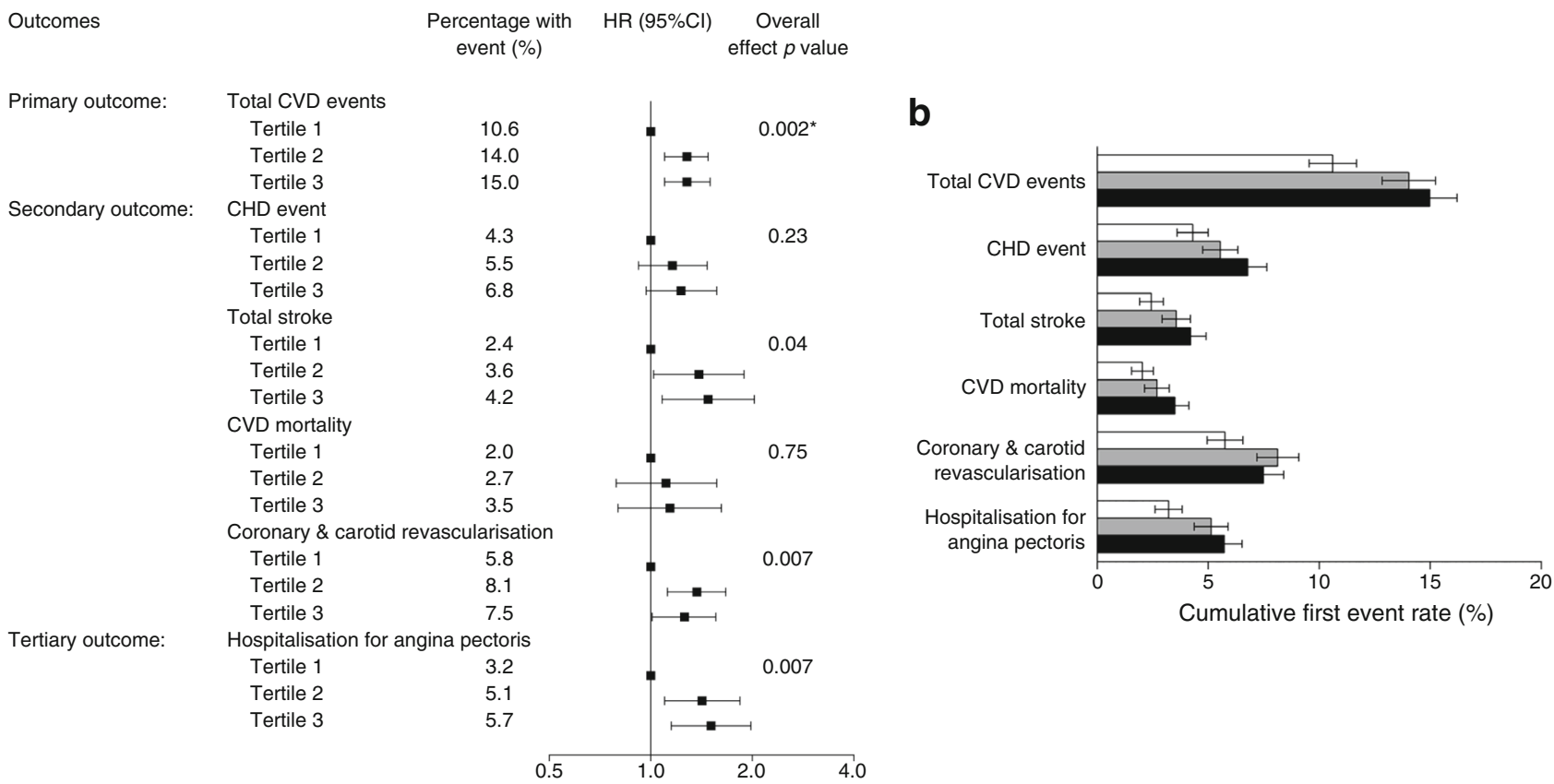

Fig. 1 Association of plasma FGF21 level at baseline with outcome events over 5 years. (a) HR values by tertile of FGF21 levels are shown for each event type. (b) Cumulative first event rates over 5 years are shown by tertile for all events. All HR and $p$ values were adjusted for age, sex, known diabetes duration, prior history of CVD, smoking (never, former and current), BMI, $\mathrm{HbA}_{1 \mathrm{c}}$, HOMA-IR, systolic BP, HDL- cholesterol, LDL-cholesterol, triacylglycerol, fibrinogen and plasma creatinine and homocysteine at baseline and treatment allocation. Error bars indicate $95 \%$ CIs. White bars, FGF21 tertile 1; grey bars, FGF21 tertile 2; black bars, FGF21 tertile $3 .{ }^{*} p<0.05$ for interaction with the treatment allocation

Table 3 Assessment of the incremental value of $\log _{\mathrm{e}}$-transformed FGF21 levels using IDI and NRI

\begin{tabular}{|c|c|c|c|c|c|c|}
\hline Outcome & IDI & $95 \% \mathrm{CI}$ & NRI $(>0)(\%)$ & $95 \% \mathrm{CI}$ & Event NRI $I^{\mathrm{a}}(\%)$ & Non-event NRI $I^{\mathrm{b}}(\%)$ \\
\hline \multicolumn{7}{|l|}{ Total CVD events } \\
\hline All participants & $0.001 *$ & $0.000,0.003$ & $10.9^{*}$ & $2.8,17.0$ & 5.4 & 5.5 \\
\hline Placebo & $0.005^{*}$ & $0.000,0.005$ & $13.8^{*}$ & $2.4,23.1$ & 9.4 & 4.3 \\
\hline Fenofibrate & 0.000 & $0.000,0.003$ & 6.3 & $-6.2,17.3$ & 0.8 & 5.5 \\
\hline CHD event & 0.000 & $0.000,0.002$ & 8.4 & $-8.5,17.9$ & 4.8 & 3.7 \\
\hline Total stroke & $0.001 *$ & $0.000,0.004$ & $17.1^{*}$ & $3.4,29.0$ & 10.7 & 6.4 \\
\hline CVD mortality & 0.001 & $0.000,0.008$ & 4.4 & $-8.7,22.7$ & -1.6 & 6.0 \\
\hline Coronary and carotid revascularisation & 0.000 & $0.000,0.002$ & 8.5 & $-4.5,15.9$ & 4.1 & 4.4 \\
\hline Hospitalisation for angina pectoris & 0.000 & $0.000,0.003$ & $12.4^{*}$ & $1.4,20.9$ & 6.4 & 6.0 \\
\hline
\end{tabular}

Comparison was made for the addition of FGF21 ( $\log _{\mathrm{e}}$-transformed) to a model containing treatment allocation, age, sex, known diabetes duration, prior history of CVD, smoking (never, former and current), BMI, HbA $1 \mathrm{c}$, HOMA-IR, systolic BP, HDL-cholesterol, LDL-cholesterol, triacylglycerol, fibrinogen and plasma creatinine and homocysteine at baseline

The category-less NRI $(>0)$ was calculated to quantify the improvement gained due to correct upward or downward change in predicted risks and was calculated as the proportion of event patients with correct upward or downward change minus incorrect upward or downward change plus the corresponding proportion among non-event patients. The IDI was calculated to measure the difference in discrimination slopes between models. The discrimination slope is the mean predicted risk of event among event patients minus the mean predicted risk of event among non-event patients and thus provides a measure of separation in predicted probabilities for event and non-event. Values above zero for the NRI and IDI indicate improved risk prediction and discrimination with the addition of FGF21 to the model

${ }^{\text {a }}$ Percentage correctly reclassified among participants who had events

${ }^{\mathrm{b}}$ Percentage correctly reclassified among participants who did not have events

$* p<0.05$ 
Discrimination and reclassification The addition of FGF21 level to a model adjusted for traditional cardiovascular risk factors resulted in a modest, but significant, increase in the Cstatistic for hospitalisation for angina pectoris from 0.692 to $0.698(p=0.03)$, but no increase was seen for the other outcomes (ESM Table 8). The IDI analysis showed a modest, but significant, improvement for total CVD events and total stroke (Table 3) after addition of FGF21 level. When assessing the reclassification using the category-free NRI, the addition of FGF21 levels to a model with traditional cardiovascular risk factors resulted in a significant increase in the NRI for total CVD events, total stroke and hospitalisation for angina pectoris (Table 3 ). In all these analyses, similar results were obtained when tertiles of FGF21 levels were used in the models (data not shown).

Association of baseline FGF21 levels with total CVD events in different subgroups As the outcome of total CVD events had the largest number of cases, a post hoc subgroup analysis was then performed according to some of the clinical characteristics, including glycaemic and lipid profiles, among the placebo group. As shown in ESM Table 9, the association of tertiles of plasma FGF21 level with total CVD events did not show any significant interaction with age, sex, previous history of CVD, BMI, smoking, known diabetes duration, $\mathrm{HbA}_{1 \mathrm{c}}$, HDLcholesterol, LDL-cholesterol, triacylglycerol or estimated GFR (all $p$ for interaction $>0.10$ ). An interaction was demonstrated with total cholesterol, in which the association tended to be stronger in patients with higher total cholesterol ( $p$ for interaction $=0.009$ ).

\section{Discussion}

FGF21 is a metabolic hormone that is produced predominantly in the liver [4] and is also produced and secreted by cardiac cells [15]. In a recent study, mice lacking FGF21 showed enhanced cardiac hypertrophy, while treatment with FGF21 protected against the hypertrophic response [15]. In another recent study, FGF21 administration was shown to mitigate myocardial infarction in response to experimental myocardial ischaemia in mice [16]. The present study is the first report of the association of plasma FGF21 level with different cardiovascular outcome events in a large-scale, well-designed clinical trial conducted in patients with type 2 diabetes.

In this 5 year follow-up study, plasma FGF21 levels at baseline were associated with total CVD events and also with coronary and carotid revascularisation. Similar trends were seen for other outcomes, although these associations lost their significance after adjusting for multiple other baseline characteristics. The findings were generally similar in both treatment arms of the study (fenofibrate vs placebo). This is consistent with previous reports showing that FGF21 levels are elevated in different human metabolic disease states, such as obesity, the metabolic syndrome and type 2 diabetes, even though FGF21 shows anti-inflammatory, hypoglycaemic and hypolipidaemic properties in animal studies [4]. In an earlier crosssectional study, serum FGF21 level was higher in 135 patients with CHD compared with the level in 35 control individuals [9]. In two other recent cross-sectional studies, higher serum FGF21 was associated with higher risk of coronary artery disease [31] and carotid atherosclerosis [32], both independently of other traditional cardiovascular risk factors. In a recent small study of 87 patients with type 2 diabetes, higher serum FGF21 levels were associated with a higher risk of the combined endpoints of cardiovascular morbidity and mortality during a 24 month follow-up period [33]. In another recent study of 60 individuals with coronary artery disease and 129 BMImatched individuals without coronary artery disease, serum FGF21 level was not associated with current coronary artery status, but was associated with hypertriacylglycerolaemia, hyperinsulinaemia, pericardial fat accumulation and the metabolic syndrome, independently of obesity [34]. The elevation in FGF21 has been suggested to be due to FGF21 resistance (as a result of impaired FGF21 signalling) or it may be a compensatory response [4]. Our study is the largest prospective study to assess the association of FGF21 levels at baseline with different cardiovascular events.

For total CVD events, the overall association of FGF21 level at baseline was also found separately in the placebo group, but not the fenofibrate group, although such difference by treatment only reached statistical significance when assessing the FGF21 level as a tertile variable and not as a $\log _{\mathrm{e}}$-transformed variable. We previously reported that fenofibrate treatment increased FGF21 levels by $85 \%$ over 5 years, relative to placebo, in a pilot study of 216 patients from the FIELD trial [22]. In the present study, fenofibrate treatment was also found to increase FGF21 levels to a similar extent (i.e. $81 \%$ ) relative to placebo over 1 year. In our previous study, the change in FGF21 levels during 5 years of fenofibrate treatment tended to be greater in patients with lower FGF21 levels at baseline [22]. This differential increase in FGF21 levels according to baseline FGF21 levels in the fenofibrate group may thus confound the association of baseline FGF21 levels with outcome events. Nevertheless, further studies are needed to elucidate the non-significant association in the fenofibrate-treated group.

In this study, we assessed the incremental value of the addition of FGF21 levels in a risk prediction model with established risk factors using C-statistics, IDI and categoryfree NRI. The results of these analyses suggested that plasma FGF21 levels could provide incremental information for total CVD events and that FGF21 may be a potential biomarker for risk assessment of some CVD events. However, interpretation should be made cautiously as the improvement in C-statistics 
and IDI was only modest and there was no outcome event that showed significant positive results in all three analyses. Nevertheless, for the events other than the total CVD event outcome, the numbers of events and hence power for analyses was substantially lower, and the small incremental value seen here is typical of models that already consist of strong and well-established risk factors $[35,36]$.

In a subgroup analysis, among the placebo group of the FIELD study, the association of FGF21 with total CVD events did not differ significantly by most clinical characteristics, except total cholesterol. This suggests that FGF21 could be a potential cardiovascular biomarker for patients with type 2 diabetes regardless of their glycaemic control or the presence of other cardiovascular risk factors such as previous history of CVD, obesity or smoking. It is interesting that the association of FGF21 with total CVD events tended to be stronger in patients with higher total cholesterol. In fact, recent studies have suggested that FGF21 may play a role in cholesterol metabolism [37-39].

FGF21 has demonstrated favourable metabolic and cardioprotective properties in animal studies [1, 5, 6, 15, 16] as well as in a recent proof-of-concept human clinical trial [14]. This may seem contradictory as higher baseline FGF21 levels were associated with higher CVD risk. It does, however, indicate that an elevated circulating level of FGF21 may be implicated as a potential biomarker for the early detection of different cardiometabolic disorders [4]. The elevation of FGF21 could be a compensatory protective response to underlying metabolic stress or could be due to FGF21 resistance as a result of impaired FGF21 signalling, which implies the need for supraphysiological doses of FGF21 to achieve therapeutic efficacy [4]. In the proof-of-concept human clinical trial, the circulating levels of the FGF21 variant, LY2405319, were 30to 300 -fold higher than the physiological levels of plasma FGF21 [14]. It may also seem contradictory as some CVD risk factors, such as longer known diabetes duration, higher $\mathrm{HbA}_{1 \mathrm{c}}$, LDL-cholesterol and fibrinogen levels and use of insulin medication, were associated with lower, not higher, FGF21 levels. The reason for this is uncertain. However, previous studies have also reported the association of higher FGF21 levels with lower LDL-cholesterol levels in pregnancy [40] and in patients with chronic kidney disease [41, 42]. In another study, patients with type 2 diabetes who had higher FGF21 levels had shorter diabetes duration than patients with lower FGF21 levels although the difference did not reach statistical significance [33]. These findings indicate that further clinical studies are needed to elucidate the systemic role and regulation of FGF21 levels in CVD in patients with type 2 diabetes.

Our study has the advantage of making use of the FIELD study, which is well designed and has good quality control, a large sample size, standardised assessments of different clinical characteristics and outcome events and availability of data on many different outcome events. However, our study also has several limitations. In this analysis, different outcomes were assessed at the same time and thus there may be a chance of obtaining false-positive results due to multiple testing. However, these outcome events were all pre-specified in the FIELD study, adjudicated by a committee masked to study treatment allocation. The analysis of different outcome events also allowed us to gain further understanding of the role of FGF21 in cardiovascular outcomes in people with type 2 diabetes. As all the patients in the FIELD study had type 2 diabetes at baseline, the findings from this study may not be generalisable to healthy people or to those at high risk of CVD but who do not have diabetes. This is particularly important because patients with type 2 diabetes have been reported to have elevated FGF21 levels [4, 10, 43]. Further studies in different clinical settings, or in participants with different characteristics, are needed to confirm our findings from the FIELD study.

In summary, higher plasma FGF21 levels at baseline were associated with higher risk of cardiovascular outcomes in the FIELD study and could be a compensatory protective response to underlying metabolic stress or impaired FGF21 signalling leading to FGF21 resistance. Studies of treatments aimed at modifying FGF21 levels and that may influence cardiovascular risk would be justified.

Acknowledgements We thank L. Buizen (National Health and Medical Research Council Clinical Trials Centre, University of Sydney, Sydney, NSW, Australia) for his assistance with the analysis of IDI and validation of some statistical analyses.

Funding The measurement of FGF21 levels in the FIELD samples was supported by a Grant-in-Aid (G 12S 6681) from the National Heart Foundation of Australia. KLO was supported by Program grants (482800 and 1037903) from the National Health and Medical Research Council (NHMRC) of Australia, and the Vice-Chancellor's Postdoctoral Fellowship from the University of New South Wales. ACK was supported by an NHMRC Program grant (1037786) and an NHMRC Fellowship grant (1024105).

Duality of interest Fournier Pharma (now part of Abbott Pharmaceuticals) sponsored the FIELD trial but had no role in data collection or analysis. KLO has received consulting fees and has served as advisory panel member for Pfizer. AJJ has served as an advisory panel member for Medtronic, has served speaker bureau for Abbott, Animaas and Medtronic and has received research support from Eli Lilly, Medtronic, Novo and Sanofi-Aventis. DRS has received research support from Amgen, Amarin, AstraZeneca and MSD and has received consultancy / educational grants from Abbott, Amgen, MSD and Janssen-Cilag. PJB has served as advisory panel member for Amgen, AstraZeneca, Eli Lilly, Kowa, MSD, Novartis, Roche and Pfizer, has served as a consultant for CSL Behring, Dezima and MSD, has received research support from MSD and Pfizer and has received honoraria for lectures from Amgen, AstraZeneca, Kowa, MSD and Pfizer. ACK has served as an Advisory Board member for Abbott Pharmaceuticals, Amgen and AstraZeneca and has received speaker and/or advisor honoraria and research support from Abbott, Amgen, Astra-Zeneca, Bristol-Myers Squibb, Eli Lilly, Merck, Novartis, Pfizer, Roche Diagnostics and Solvay. KAR has served as a consultant for CSL Behring and Regeneron. All other authors declare that there is no duality of interest associated with their contribution to this manuscript. 
Contribution statement KLO wrote the first draft of the manuscript. $\mathrm{KLO}, \mathrm{ACK}$ and KAR contributed to conception and design, data analysis and interpretation of data. KLO, ASJ, AJJ, AX, DRS, PJB, RSS, MRT and ACK contributed to the acquisition of data. KLO, RO and WTH contributed to the data analysis. ASJ, ACK, and KAR supervised the studies. KLO, AJJ, DRS, PJB, ACK and KAR contributed to the obtaining of funding. All authors reviewed and edited the manuscript. All authors gave final approval of the version to be published. KLO is responsible for the integrity of the work as a whole.

\section{References}

1. Kharitonenkov A, Shiyanova TL, Koester A et al (2005) FGF-21 as a novel metabolic regulator. J Clin Invest 115:1627-1635

2. Badman MK, Pissios P, Kennedy AR, Koukos G, Flier JS, MaratosFlier E (2007) Hepatic fibroblast growth factor 21 is regulated by PPARalpha and is a key mediator of hepatic lipid metabolism in ketotic states. Cell Metab 5:426-437

3. Inagaki T, Dutchak P, Zhao G et al (2007) Endocrine regulation of the fasting response by PPARalpha-mediated induction of fibroblast growth factor 21. Cell Metab 5:415-425

4. Woo YC, Xu A, Wang Y, Lam KS (2013) Fibroblast growth factor 21 as an emerging metabolic regulator: clinical perspectives. Clin Endocrinol (Oxf) 78:489-496

5. Wente W, Efanov AM, Brenner M et al (2006) Fibroblast growth factor-21 improves pancreatic beta-cell function and survival by activation of extracellular signal-regulated kinase $1 / 2$ and Akt signaling pathways. Diabetes 55:2470-2478

6. Kharitonenkov A, Wroblewski VJ, Koester A et al (2007) The metabolic state of diabetic monkeys is regulated by fibroblast growth factor-21. Endocrinology 148:774-781

7. Zhang X, Yeung DC, Karpisek M et al (2008) Serum FGF21 levels are increased in obesity and are independently associated with the metabolic syndrome in humans. Diabetes 57:1246-1253

8. Li H, Fang Q, Gao F et al (2010) Fibroblast growth factor 21 levels are increased in nonalcoholic fatty liver disease patients and are correlated with hepatic triglyceride. J Hepatol 53:934-940

9. Lin Z, Wu Z, Yin X et al (2010) Serum levels of FGF-21 are increased in coronary heart disease patients and are independently associated with adverse lipid profile. PLoS One 5:e15534

10. Chen C, Cheung BM, Tso AW et al (2011) High plasma level of fibroblast growth factor 21 is an independent predictor of type 2 diabetes: a 5.4-year population-based prospective study in Chinese subjects. Diabetes Care 34:2113-2115

11. Wu AL, Kolumam G, Stawicki S et al (2011) Amelioration of type 2 diabetes by antibody-mediated activation of fibroblast growth factor receptor 1. Sci Transl Med 3:113ra126

12. Foltz IN, Hu S, King C et al (2012) Treating diabetes and obesity with an FGF21-mimetic antibody activating the $\beta$ Klotho/FGFR1c receptor complex. Sci Transl Med 4:162ra153

13. Mu J, Pinkstaff J, Li Z et al (2012) FGF21 analogs of sustained action enabled by orthogonal biosynthesis demonstrate enhanced antidiabetic pharmacology in rodents. Diabetes 61:505-512

14. Gaich G, Chien JY, Fu H et al (2013) The effects of LY2405319, an FGF21 analog, in obese human subjects with type 2 diabetes. Cell Metab 18:333-340

15. Planavila A, Redondo I, Hondares E et al (2013) Fibroblast growth factor 21 protects against cardiac hypertrophy in mice. Nat Commun 4:2019

16. Liu SQ, Tefft BJ, Roberts DT et al (2012) Cardioprotective proteins upregulated in the liver in response to experimental myocardial ischemia. Am J Physiol Heart Circ Physiol 303:H1446-H1458
17. FIELD Study Investigators (2004) The need for a large-scale trial of fibrate therapy in diabetes: the rationale and design of the Fenofibrate Intervention and Event Lowering in Diabetes (FIELD) study. [ISRCT N64783481]. Cardiovasc Diabetol 3:9

18. Keech A, Simes RJ, Barter P et al (2005) Effects of long-term fenofibrate therapy on cardiovascular events in 9795 people with type 2 diabetes mellitus (the FIELD study): randomised controlled trial. Lancet 366:1849-1861

19. Rajamani K, Colman PG, Li LP et al (2009) Effect of fenofibrate on amputation events in people with type 2 diabetes mellitus (FIELD study): a prespecified analysis of a randomised controlled trial. Lancet 373:1780-1788

20. Keech AC, Mitchell P, Summanen PA et al (2007) Effect of fenofibrate on the need for laser treatment for diabetic retinopathy (FIELD study): a randomised controlled trial. Lancet 370:1687-1697

21. Burgess DC, Hunt D, Li L et al (2010) Incidence and predictors of silent myocardial infarction in type 2 diabetes and the effect of fenofibrate: an analysis from the Fenofibrate Intervention and Event Lowering in Diabetes (FIELD) study. Eur Heart J 31:92-99

22. Ong KL, Rye KA, O'Connell R et al (2012) Long-term fenofibrate therapy increases fibroblast growth factor 21 and retinol-binding protein 4 in subjects with type 2 diabetes. J Clin Endocrinol Metab 97:4701-4708

23. Scott R, Best J, Forder P et al (2005) Fenofibrate Intervention and Event Lowering in Diabetes (FIELD) study: baseline characteristics and short-term effects of fenofibrate [ISRCTN64783481]. Cardiovasc Diabetol 4:13

24. Taskinen MR, Sullivan DR, Ehnholm C et al (2009) Relationships of HDL cholesterol, ApoA-I, and ApoA-II with homocysteine and creatinine in patients with type 2 diabetes treated with fenofibrate. Arterioscler Thromb Vasc Biol 29:950-955

25. Levey AS, Bosch JP, Lewis JB, Greene T, Rogers N, Roth D (1999) A more accurate method to estimate glomerular filtration rate from serum creatinine: a new prediction equation. Modification of Diet in Renal Disease Study Group. Ann Intern Med 130:461-470

26. Pasta D (2009) Learning when to be discrete: continuous vs. categorical predictors. SAS Glob Forum Pap 248:1-10

27. Newson RB (2011) Comparing the predictive powers of survival models using Harrell's C or Somers' D. Stata J 10:339-358

28. Pencina MJ, D'Agostino RB Sr, D’Agostino RB Jr, Vasan RS (2008) Evaluating the added predictive ability of a new marker: from area under the ROC curve to reclassification and beyond. Stat Med 27: $157-172$

29. Pencina MJ, D'Agostino RB Sr, Steyerberg EW (2011) Extensions of net reclassification improvement calculations to measure usefulness of new biomarkers. Stat Med 30:11-21

30. May S, Hosmer DW (1998) A simplified method of calculating an overall goodness-of-fit test for the Cox proportional hazards model. Lifetime Data Anal 4:109-120

31. Shen Y, Ma X, Zhou J et al (2013) Additive relationship between serum fibroblast growth factor 21 level and coronary artery disease. Cardiovasc Diabetol 12:124

32. Chow WS, Xu A, Woo YC et al (2013) Serum fibroblast growth factor-21 levels are associated with carotid atherosclerosis independent of established cardiovascular risk factors. Arterioscler Thromb Vasc Biol 33:2454-2459

33. Lenart-Lipińska M, Matyjaszek-Matuszek B, Gernand W, Nowakowski A, Solski J (2013) Serum fibroblast growth factor 21 is predictive of combined cardiovascular morbidity and mortality in patients with type 2 diabetes at a relatively short-term follow-up. Diabetes Res Clin Pract 101:194-200

34. Lee Y, Lim S, Hong ES, Kim JH et al (2014) Serum FGF21 concentration is associated with hypertriglyceridaemia, hyperinsulinaemia and pericardial fat accumulation, independently of obesity, but not with current coronary artery status. Clin Endocrinol (Oxf) 80:57-64 
35. de Backer G, Graham I, Cooney MT (2012) Do novel biomarkers add to existing scores of total cardiovascular risk? Eur J Prev Cardiol 19(2 Suppl):14-17

36. Ahluwalia N, Blacher J, Szabo de Edelenyi F et al (2013) Prognostic value of multiple emerging biomarkers in cardiovascular risk prediction in patients with stable cardiovascular disease. Atherosclerosis 228:478-484

37. Kosola S, Lampela H, Gylling H et al (2012) Cholesterol metabolism altered and FGF21 levels high after pediatric liver transplantation despite normal serum lipids. Am J Transplant 12:2815-2824

38. Habegger KM, Stemmer K, Cheng C et al (2013) Fibroblast growth factor 21 mediates specific glucagon actions. Diabetes 62:1453-1463

39. Lin XL, He XL, Zeng JF et al (2014) FGF21 increases cholesterol efflux by upregulating ABCA1 through the ERK1/2-PPAR $\gamma-\mathrm{LXR} \alpha$ pathway in THP1 macrophage-derived foam cells. DNA Cell Biol 33:514-521

40. Stepan H, Kley K, Hindricks J et al (2013) Serum levels of the adipokine fibroblast growth factor-21 are increased in preeclampsia. Cytokine 62:322-326

41. Lin Z, Zhou Z, Liu Y et al (2011) Circulating FGF21 levels are progressively increased from the early to end stages of chronic kidney diseases and are associated with renal function in Chinese. PLoS One 6:e18398

42. Hindricks J, Ebert T, Bachmann A et al (2014) Serum levels of fibroblast growth factor-21 are increased in chronic and acute renal dysfunction. Clin Endocrinol (Oxf) 80:918-924

43. Kralisch S, Tönjes A, Krause K et al (2013) Fibroblast growth factor21 serum concentrations are associated with metabolic and hepatic markers in humans. J Endocrinol 216:135-143 